\title{
Development of a novel immune-related IncRNA signature as a prognostic classifier for endometrial carcinoma
}

\author{
Jinhui $\operatorname{Liu}^{1 *}$, Jie $\mathrm{Mei}^{2^{*}}$, Yichun Wang $3^{3^{*}}$, Xucheng Chen ${ }^{4}$, Jiadong Pan², Laigen Tong ${ }^{5 凶}$ and Yan Zhang ${ }^{6 \bowtie}$ \\ 1. Department of Gynecology, The First Affiliated Hospital of Nanjing Medical University, Nanjing 210029, Jiangsu, China. \\ 2. Wuxi School of Clinical Medicine, Nanjing Medical University, Wuxi 214023, Jiangsu, China \\ 3. Department of Urology, The First Affiliated Hospital of Nanjing Medical University, Nanjing 210029, Jiangsu, China. \\ 4. College of Pharmacy, Nanjing Medical University, Nanjing 211166, Jiangsu, China. \\ 5. Department of Hematology, Yixing People's Hospital, The Affiliated Hospital of Jiangsu University, Yixing 214200, Jiangsu, China. \\ 6. Department of Gynecology and Obstetrics, Wuxi Maternal and Child Health Hospital, the Affiliated Hospital of Nanjing Medical University, Wuxi 214000, Jiangsu, \\ China. \\ *These authors contributed equally to this work.
}

$\triangle$ Corresponding authors: Yan Zhang, M.D. Department of Gynecology and Obstetrics, Wuxi Maternal and Child Health Hospital, the Affiliated of Nanjing Medical University. No. 48, Huaishu Road, Wuxi 214000, Jiangsu, China. E-mail: fuyou2007@126.com; Laigen Tong, M.Sc. Department of Hematology, Yixing People's Hospital, the Affiliated Hospital of Jiangsu University. No. 75, Zhenguan Road, Yixing 214200, Jiangsu, China. E-mail: dr_tonglaigen2@126.com.

(C) The author(s). This is an open access article distributed under the terms of the Creative Commons Attribution License (https://creativecommons.org/licenses/by/4.0/). See http://ivyspring.com/terms for full terms and conditions.

Received: 2020.07.28; Accepted: 2020.12.06; Published: 2021.01.01

\begin{abstract}
Endometrial carcinoma $(\mathrm{EnCa})$ is one of the deadliest gynecological malignancies. The purpose of the current study was to develop an immune-related IncRNA prognostic signature for EnCa. In the current research, a series of systematic bioinformatics analyses were conducted to develop a novel immune-related IncRNA prognostic signature to predict disease-free survival (DFS) and response to immunotherapy and chemotherapy in EnCa. Based on the newly developed signature, immune status and mutational loading between high- and low-risk groups were also compared. A novel 13-IncRNA signature associated with DFS of EnCa patients was ultimately developed using systematic bioinformatics analyses. The prognostic signature allowed us to distinguish samples with different risks with relatively high accuracy. In addition, univariate and multivariate Cox regression analyses confirmed that the signature was an independent factor for predicting DFS in EnCa. Moreover, a predictive nomogram combined with the risk signature and clinical stage was constructed to accurately predict 1-, 2-, 3-, and 5-year DFS of EnCa patients. Additionally, EnCa patients with different levels of risk had markedly different immune statuses and mutational loadings. Our findings indicate that the immune-related 13-IncRNA signature is a promising classifier for prognosis and response to immunotherapy and chemotherapy for EnCa.
\end{abstract}

Key words: immune-related lncRNA; endometrial carcinoma; signature; bioinformatics

\section{Introduction}

Endometrial carcinoma (EnCa), which originates from glandular epithelial cells of the endometrium, is the third most common type of malignant tumor in the female reproductive system worldwide [1]. According to the statistical data published by the International Agency for Research on Cancer, EnCa comprises $4.8 \%$ of cancers and has a tumor-related mortality rate of $2.1 \%$ among females worldwide [2]. According to the International Federation of Gynecology and Obstetrics (FIGO) criteria, EnCa is divided into two subtypes based on endocrine and clinical features: type I carcinomas, which are related to endometrial hyperplasia and are estrogen dependent, and type II carcinomas, which are related to endometrial atrophy and are estrogen independent [3]. Although continual efforts have been made to overcome one difficulty after another in the long-term quest to treat EnCa, the prognosis of patients with advanced-stage disease is unsatisfactory. Therefore, it is of great significance to identify novel strategies to accurately predict the prognosis of EnCa.

Recently, it was proven that immunotherapies can be novel therapeutic strategies for EnCa and that the local immune status affects tumor progression and determines the response to immunotherapies [4,5]. Long noncoding RNAs (lncRNAs) have a wide range 
of functional activities [6, 7], and dysregulation of critical lncRNAs may contribute to the carcinogenesis and progression of EnCa [8, 9]. Moreover, lncRNAs have been identified as crucial nodes in regulating tumor immunity in multiple cancers [10, 11]. For example, lncMX1-215 markedly downregulates IFNainduced programmed cell death 1 ligand 1 (PD-L1) and galectin-9 expression by interrupting GCN5/ H3K27ac binding in head and neck squamous cell carcinoma [12]. Therefore, dysregulation of immunerelated lncRNAs may be identified as potential therapeutic targets and have prognostic value for EnCa patients.

In the current research, we developed a novel immune-related 13-lncRNA signature for EnCa patients that could accurately predict disease-free survival (DFS). In addition, based on the signature, the immune status and tumor mutation burden (TMB) in EnCa samples from different groups were distinctly varied. Notably, the findings obtained from this research may contribute to providing potential foundations for subsequent in-depth immune-related studies, to provide new insights into the individualized treatment of EnCa.

\section{Methods}

\section{TCGA data acquisition}

Level-3 mRNA expression profiles and clinical data were collected from 552 EnCa and 23 normal samples in the TCGA database (https:// cancergenome.nih.gov). DFS was defined as the time from the starting point to disease recurrence or death due to disease progression, which was treated as the end point of observation in this research. After integration of clinical information, a total of 481 samples were defined as an entire set, which was randomly divided into a training set and a testing set. The testing set with 240 samples and the entire set were used to verify findings obtained from the training set with 241 samples. Both the entire mRNA profile data and the clinical information of the samples are publicly available.

\section{Definition of immune-related IncRNAs}

First, immune-related genes were obtained from the Molecular Signatures Database v4.0 (Immune system process M13664, Immune response M19817, http://www.broadinstitute.org/gsea/msigdb/index. jsp) [13]. In addition, the lncRNA profile was extracted from mRNA expression data on the basis of the GENCODE project (http://www.gencodegenes. org) [14]. Then, a cohort of immune-related lncRNAs was identified according to Pearson correlation analysis, which was defined as lncRNA whose expression is correlated with any immune-related genes (at least one gene) $(|\mathrm{R}|>0.5, P<0.0001)$.

\section{Construction of immune-related the IncRNA signature for $\mathrm{EnCa}$}

These samples were randomly classified into the training cohort $(\mathrm{n}=241)$ and the testing cohort $(\mathrm{n}=$ 240). The training cohort was used for prognostic signature construction, while the testing cohort and entire cohort were used for validation of the signature. Univariate Cox regression analysis was applied to screen prognosis-related genes from 45 immune-related lncRNAs with the criteria of $P \leq 0.05$. LASSO Cox analysis was applied to identify lncRNAs most associated with DFS, and 10-round crossvalidation was conducted to prevent overfitting. The filtered genes were entered into the multivariate Cox regression analysis, and a scoring model was built to predict DFS.

The risk score for each patient was then calculated using the following formula: risk score $=$ -0.970896 * AL080317.2 + $0.173290 *$ ERICH6-AS1 1.091183 * AC016877.3 + $0.560300 *$ MCCC1-AS1 +0.153787 * AC120053.1 + 0.383809 * AC138932.5 0.690101 * ZNF433-AS1 - 0.123376 * SCARNA9 0.344099 * DBH-AS1 + 0.622167 * AL157932.1 + $0.236900 *$ AC073046.1 - $1.039016 *$ POC1B-AS1 + 0.428151 * AP003419.3. EnCa patients were divided into a high-risk group and a low-risk group at the cutoff of the median risk score. Univariate and multivariate Cox regression analysis was used to assess the prognostic values of risk score and other clinicopathological characteristics.

\section{Estimation of tumor-infiltrating immune cells}

Transcriptome expression with standard annotation was uploaded to the CIBERSORT website (http://cibersort.stanford.edu/) [15], which was applied to assess the proportions of 22 types of tumorinfiltrating immune cells using the CIBERSORT algorithm following the standard procedure.

\section{Construction of mRNA-IncRNA coexpression networks}

We analyzed the potential coexpression relationships of prognostic lncRNAs by Pearson's correlation analysis $(|\mathrm{R}|>0.5, P<0.0001)$. Then, the lncRNA-mRNA interaction network was visualized by Cytoscape software.

\section{Gene set enrichment analysis}

In the entire TCGA cohort, samples of EnCa were divided into two groups according to the median risk score. In the assessment of potential biological functions related to risk score, gene set enrichment analysis (GSEA) was performed to detect associated signaling pathways between the two 
groups [16]. KEGG terms enriched with $\mathrm{FDR} \leq 0.05$ were identified.

\section{Construction and validation of a predictive nomogram}

Clinical stage and risk score, which had independent prognostic value, were incorporated to construct a nomogram to evaluate the probability of 1-, 2-, 3-, and 5-year DFS in EnCa. Validation of the nomogram was evaluated by calibration plot. The calibration curve of the nomogram was plotted to assess the nomogram-predicted probabilities against the actual rates.

\section{Mutation analysis}

To compare the mutational loading between the two groups, the mutation annotation format (MAF) based on the TCGA cohort was functioned by the "maftools" package [17]. In addition, TMB between the high- and low-risk groups was compared, and the correlation between $\mathrm{TMB}$ and risk score was also assessed.

\section{The immunophenoscore analysis}

The immunophenoscore (IPS) of the tumor samples was calculated by analyzing the expression of genes in the following four major categories that determine immunogenicity: effector cells, immunosuppressive cells, MHC molecules, and immunomodulators [18]. The IPS was calculated with a range of 0-10 based on the z-score for gene expression of representative cell types. The IPS for EnCa patients was downloaded from the Cancer Immunome Atlas (TCIA, https://tcia.at/home).

\section{Chemotherapeutic response prediction}

In this study, the chemo-response to several drugs was predicted based on the online pharmaceutic database (Genomics of Drug Sensitibity in Cancer, GDSC), this database performed a largescale drug screen incorporating detailed genomic analyses to systematically identify drug response biomarkers. This database screened $>1000$ genetically characterised human cancer cell lines with a wide range of anti-cancer therapeutics. The sensitivity patterns of the cell lines are correlated with extensive genomic data to identify genetic features that are predictive of sensitivity. This large collection of cell lines enables this database to capture much of the genomic heterogeneity that underlies human cancer, and which appears to play a critical role in determining the variable response of patients to treatment with specific agents. The half-maximal inhibitory concentration (IC50) of each drug was detected in plenty of cell lines. Besides, we also used the pRRophetic R package. This R package established statistical models from gene expression and drug sensitivity data in a very large panel of cancer cell lines, then applying these models to gene expression data from primary tumor tissues. With the help of the data from GDSC and pRRophetic $\mathrm{R}$ package, we predicted the IC50 in patients. For individual patients in the TCGA group, the exact treatment scheme is different, in this study; we predicted the chemo-response of several drugs based on the genomic gene expression pattern in high-risk and low-risk groups. All these predictions were based on the online pharmaceutic database rather than the actual treatment response. All parameters were set by the default values with removal of the batch effect of "combat" and tissue type of "allSoldTumors", and duplicate gene expression was summarized as the mean value [19].

\section{Statistical analysis}

All statistical analyses were applied by $\mathrm{R}$ version 3.6.3 (R package: pheatmap, ggplot2, rms, glmnet, forest plot, survminer, survival ROC, maftools, pRRophetic). For all analyses, two-tailed $P \leq 0.05$ was regarded as statistically significant.

\section{Results}

\section{Identification of immune-related IncRNAs}

A total of 331 immune-related genes were collected from the Molecular Signatures Database. Then, 363 immune-related lncRNAs were identified according to Pearson correlation analysis $(|\mathrm{R}|>0.5, P$ $<0.0001)$.

\section{Construction and validation of the immune- related 13-IncRNA signature}

To construct an immune-related lncRNA prognostic signature, we used univariate Cox regression to screen the prognostic values of 363 lncRNAs. A total of 45 prognosis-associated lncRNAs were reserved with the criterion of $P \leq 0.05$. Next, LASSO Cox analysis with ten-fold cross-validation was performed to further narrow the effective prognosis-associated lncRNAs (Fig. S1A, B). Subsequently, multivariate Cox proportional hazards regression analysis was used to build the prognostic signature. Finally, 13 immune-related lncRNAs were used to establish a prognostic signature (Fig. S1C). The risk score for each EnCa sample was calculated according to the expression levels of 13 immunerelated lncRNAs and corresponding coefficients. Risk score $=-0.970896 *$ AL080317.2 $+0.173290 *$ ERICH6-AS1 - 1.091183 * AC016877.3 + 0.560300 * MCCC1-AS1 + 0.153787 * AC120053.1 + 0.383809 * AC138932.5 - 0.690101 * ZNF433-AS1 - 0.123376 * SCARNA9 - $0.344099 *$ DBH-AS1 + 0.622167 * 
AL157932.1 + 0.236900 * AC073046.1 - 1.039016 * POC1B-AS1 + 0.428151 * AP003419.3.

According to the median risk score, all EnCa samples were divided into high- and low-risk groups. The distribution of risk score, survival status, and expression of 13 hub lncRNAs in the training set are shown in Fig. 1A-B. Kaplan-Meier analysis suggested a remarkable difference between the two groups $(P<0.001$, Fig. 1C). In the training set, the 1-, 2-, 3-, and 5 -year AUCs were $0.668,0.878$, and 0.884 , respectively (Fig. 1D). To validate the prognostic value of this risk signature, the testing and entire cohort were used to further test the prognostic impact of the risk signature. The distributions of risk score, survival status, and expression of 13 hub lncRNAs in the testing and entire sets are shown in Fig. 2A-B and Fig. 3A-B. Similar to the training set, the clinical outcome of patients with high risk was remarkably worse than that of patients with low risk in both the testing $(P=0.005)$ and entire sets $(P<0.001)$ (Figs. 2C, 3C). Additionally, time-dependent ROC analysis demonstrated the satisfactory prognostic accuracy of the established signature in the testing and entire sets (Figs. 2D, 3D). Furthermore, among multiple clinicopathological factors, the risk score showed the largest AUC for 1-year, 3-year, and 5-year DFS (Fig. 4A-F).

\section{Expression of 13 hub IncRNAs and their association with immune cell infiltration}

A total of 13 hub lncRNAs were incorporated into the construction of the novel immune-related prognostic signature, so we next determined their expression and association with immune cell infiltration in EnCa. As shown in Fig. S2, four lncRNAs, namely, AL080317.2, ZNF433-AS1, SCARNA9, and AC073046.1, were significantly overexpressed in EnCa tissues compared with normal tissues $(P<0.05$, Fig. S2A-D). Three lncRNAs, namely, AC016877.3, AC120053.1, and AL157932.1, were downregulated in EnCa tissues ( $P<0.05$, Fig. S2E-G). However, the other six lncRNAs showed no remarkable dysregulation in tumor tissues $(P>0.05$, Fig. S3A-F). We further assessed the association between the expression of 13 hub lncRNAs and immune cell infiltration. Most lncRNAs were correlated with specific immune cell infiltration; among them, ERICH6-AS1 had the strongest correlation with immune cell infiltration (Fig. S4).
A

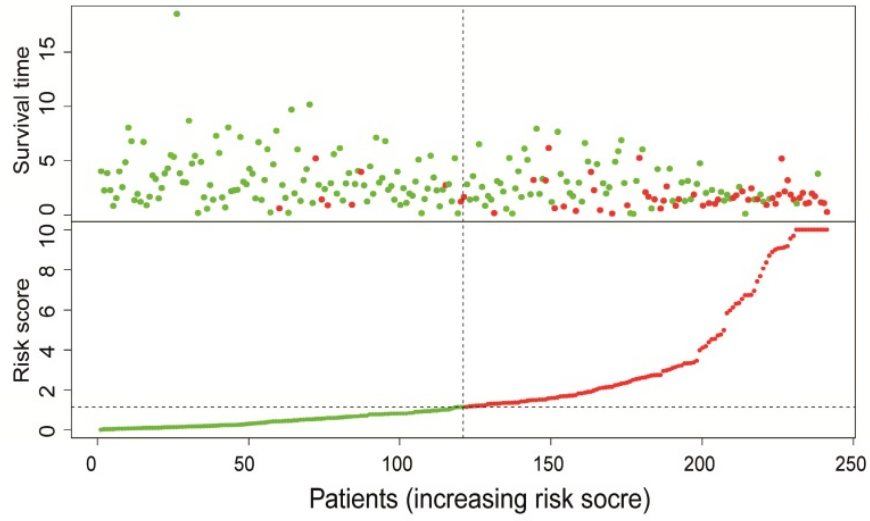

B

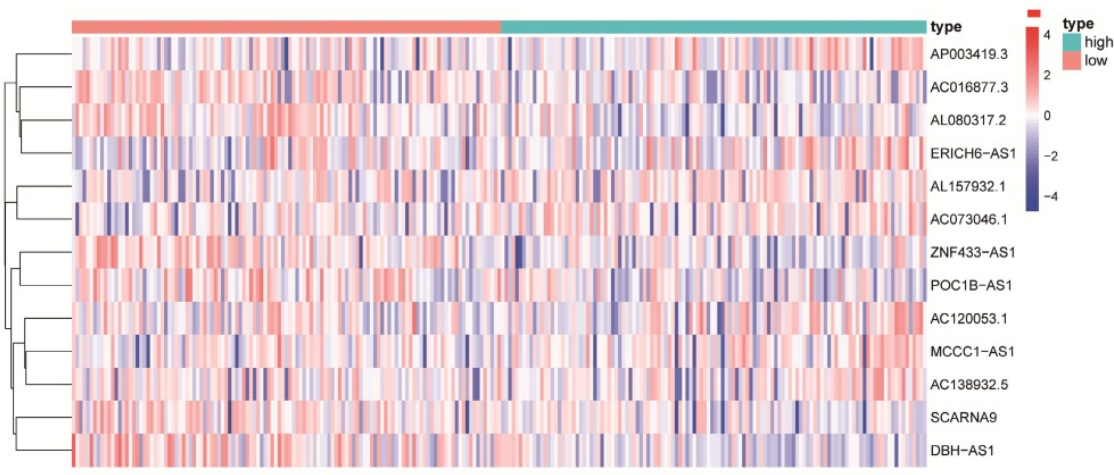

C

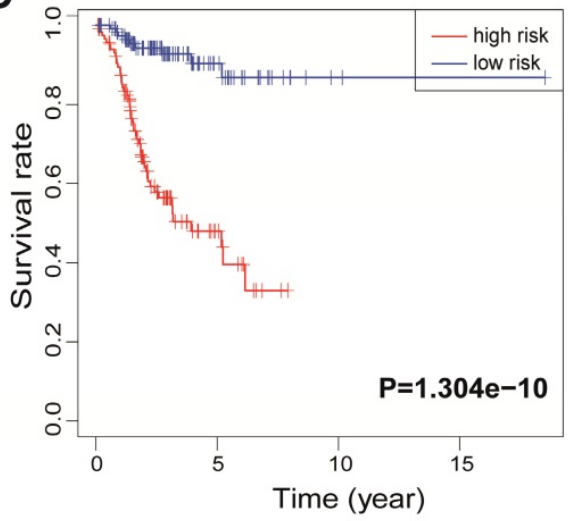

D

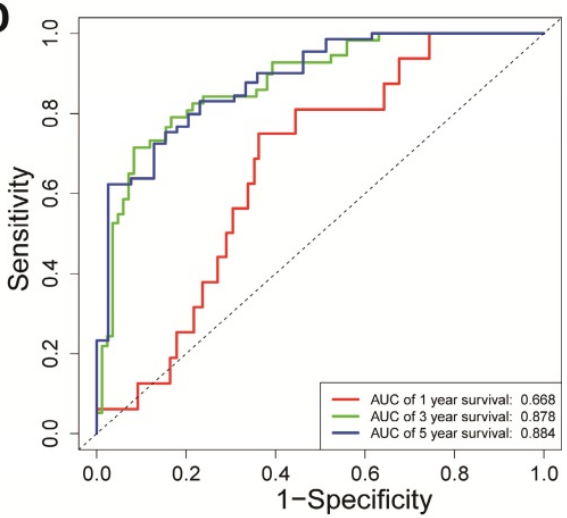

Figure 1. Identification of the immune-related IncRNA signature in the training cohort. (A) Survival status and risk score distribution in the high- and low-risk groups. Green dots: surviving patients; red dots: dead patients. (B) Expression patterns of 13 IncRNAs in high- and low-risk groups. (C) Kaplan-Meier curve analysis of DFS of EnCa patients in high- and low-risk groups. (D) Time-dependent ROC curve analysis. 
A

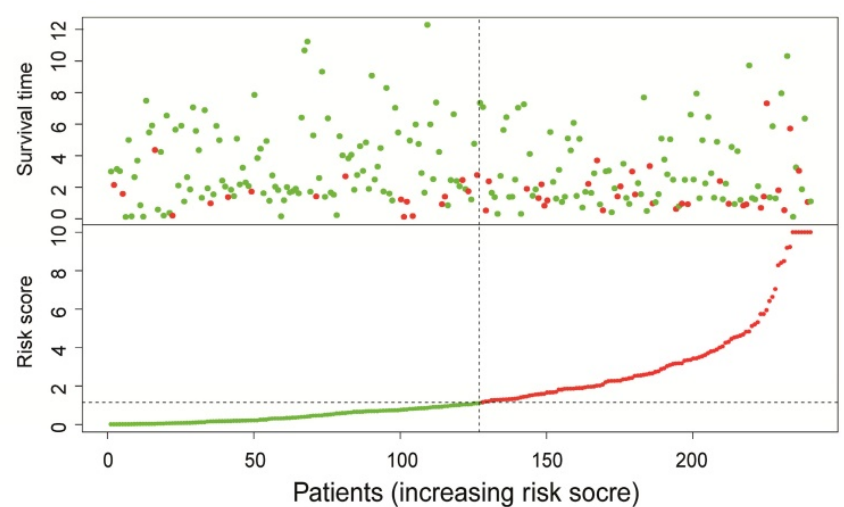

C

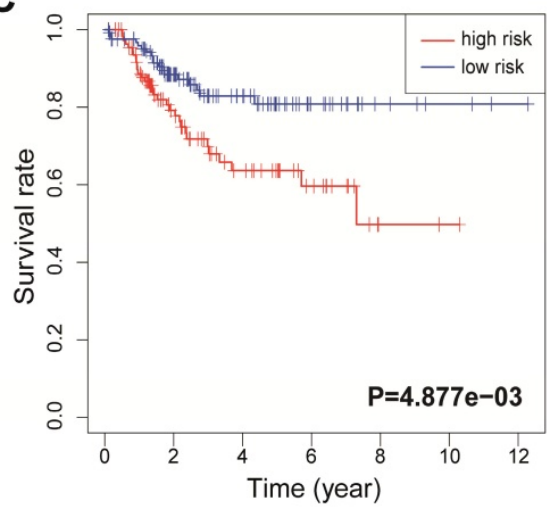

B

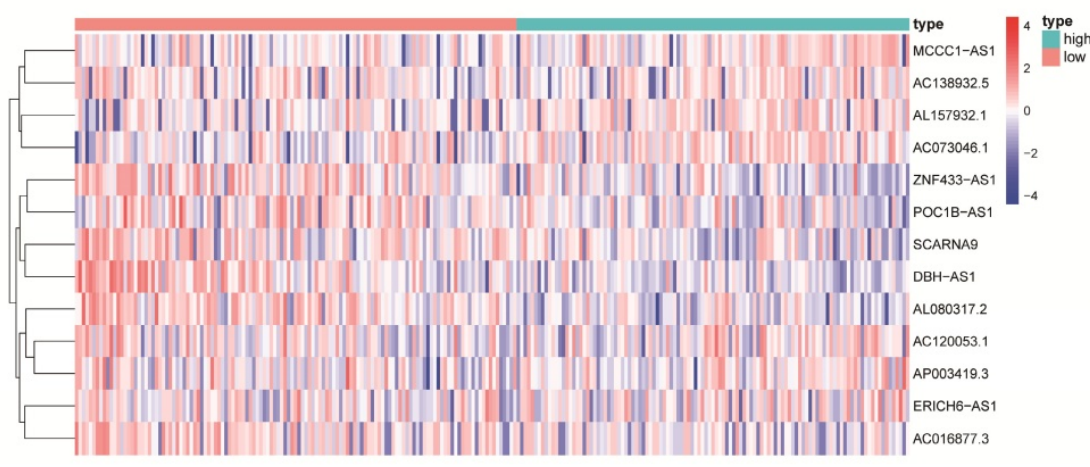

D

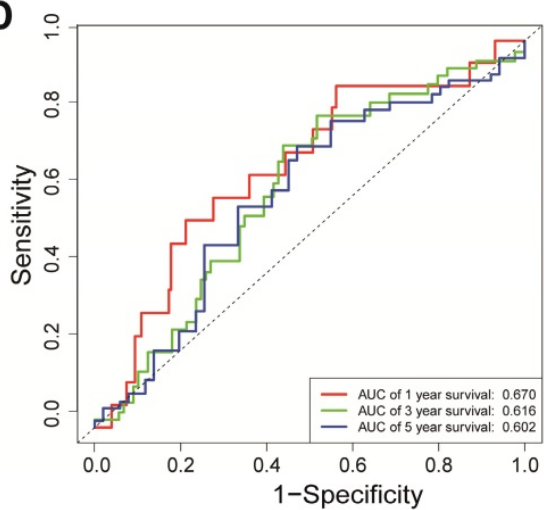

Figure 2. Validation of the immune-related IncRNA signature in the testing cohort. (A) Survival status and risk score distribution in the high- and low-risk groups. Green dots: surviving patients; red dots: dead patients. (B) Expression patterns of 13 IncRNAs in high- and low-risk groups. (C) Kaplan-Meier curve analysis of DFS of EnCa patients in highand low-risk groups. (D) Time-dependent ROC curve analysis.

Immune-related mRNAs showing a Pearson correlation of $>0.5$ with a 13-lncRNA signature were selected according to their association with lncRNAs, and altogether, 21 immune-related mRNAs were plotted into the network (Fig. S5A-B).

\section{Identification of risk score-associated biological pathways}

To define the potential biological processes associated with risk score in EnCa, GSEA was conducted to identify the enriched KEGG pathways. The results showed that "cell cycle", "ECM receptor interaction", "ERBB signaling pathway", "TGF beta signaling pathway", and "Wnt signaling pathway" were enriched in EnCa samples with high-risk scores (Fig. S6A). Since these lncRNAs were immune-related genes, the low-risk group was enriched in several immune-related pathways, including "cytokinecytokine receptor interaction" and "intestinal immune network for IgA production", which were related to immunity (Fig. S6B). In addition, "Allograft rejection", "Asthma", and "Graft versus host disease" were also enriched in EnCa samples with low-risk scores (Fig. S6B).

\section{Survival analysis of the signature in the training, testing and entire sets}

Based on the newly developed signature, we next assessed the prognostic impact of the risk signature in EnCa patients with different clinicopathological characteristics in the entire cohort. First, patients who were older (aged $>60$ years), had poor differentiation (G3\&G4), and had the mixed and serous subtype tended to have higher risk scores $(P<0.05$, Fig. S7A-C). Moreover, as shown in Fig. S8, the risk score reached satisfactory prognostic discrimination in all patients with different characteristics $(P<0.05$, Fig. S8A-D).

Univariate and multivariate Cox regression analyses were used to evaluate whether the 13-lncRNA signature was an independent prognostic indicator for EnCa patients. In the univariate Cox regression analysis, the hazard ratios (HRs) of the risk score and 95\% CIs were 1.189 (1.144-1.234), 1.060 (1.010-1.113), and 1.112 (1.083-1.141) in the training, testing, and entire sets, respectively (Fig. 5A-C). In the multivariate Cox regression analysis, the HRs of the risk score and 95\% CIs were 1.177 (1.131-1.224), 1.058 (1.001-1.119), and 1.110 (1.079-1.143) in the training, testing, and entire sets, respectively (Fig. 5D-F). 
A

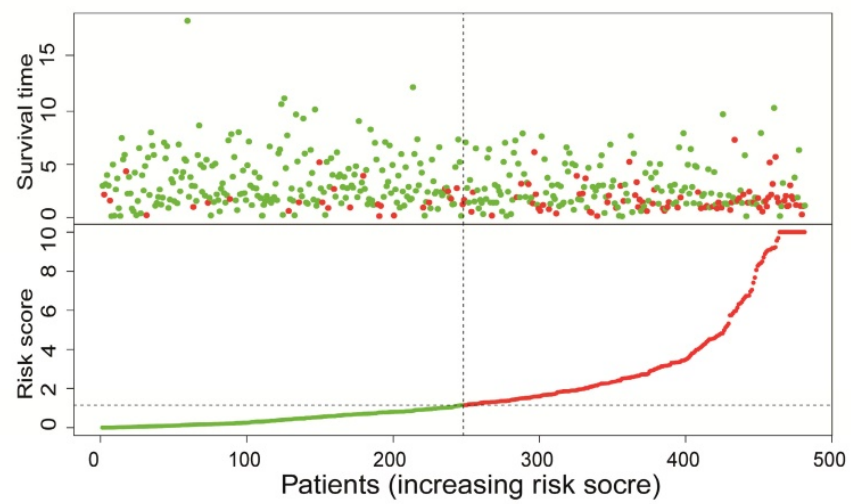

C

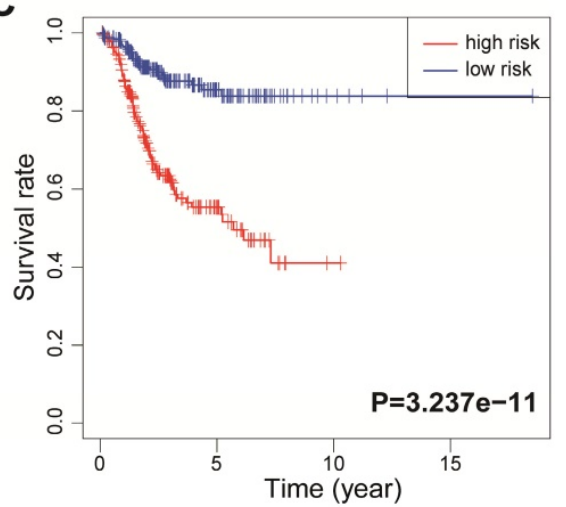

B

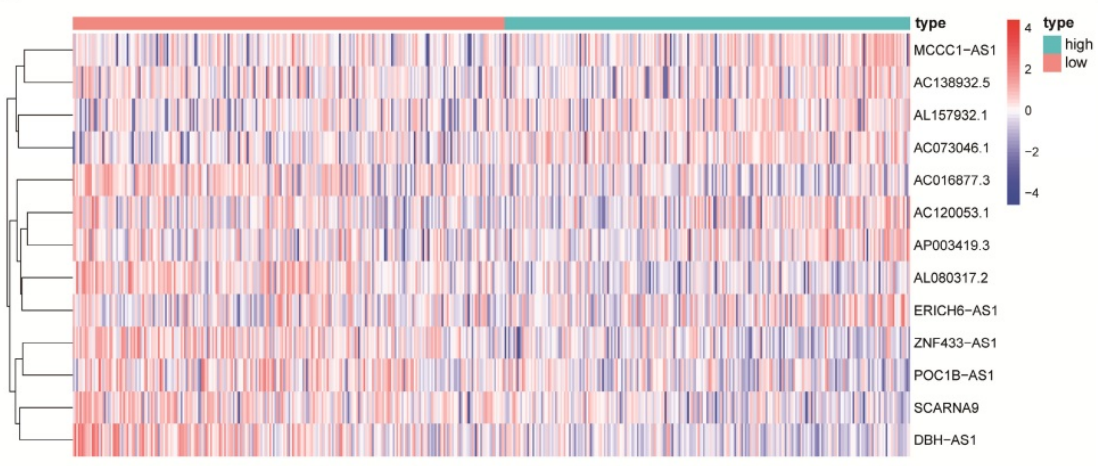

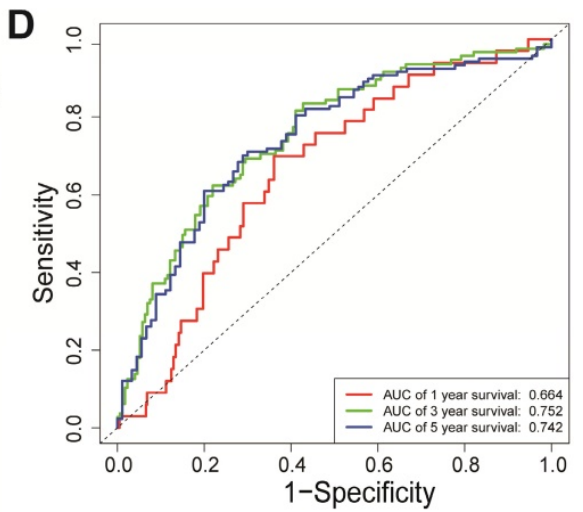

Figure 3. Validation of the immune-related IncRNA signature in the entire cohort. (A) Survival status and risk score distribution in the high- and low-risk groups. Green dots: surviving patients; red dots: dead patients. (B) Expression patterns of 13 IncRNAs in high- and low-risk groups. (C) Kaplan-Meier curve analysis of DFS of EnCa patients in highand low-risk groups. (D) Time-dependent ROC curve analysis.

A

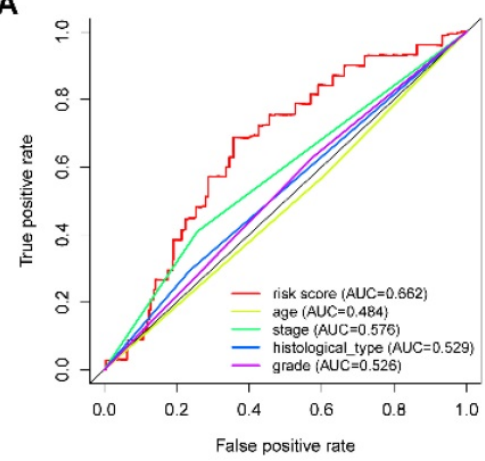

D

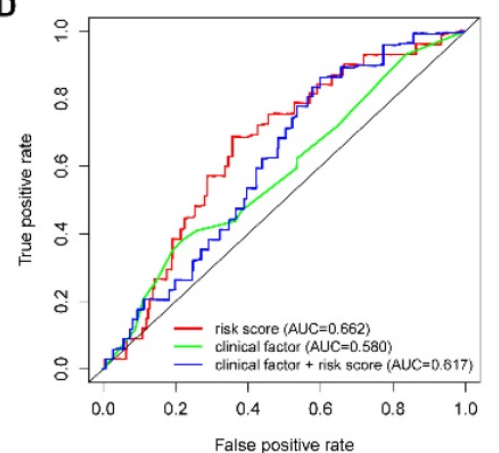

B

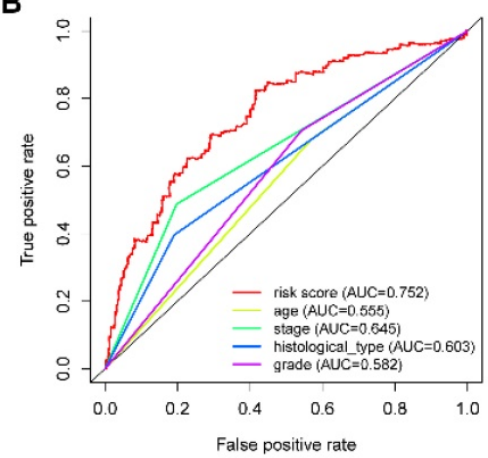

E

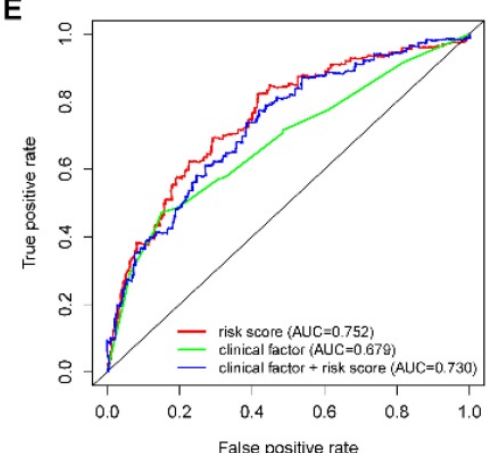

C

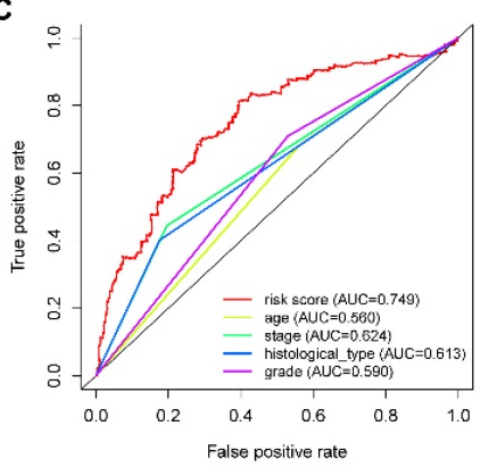

$\mathbf{F}$

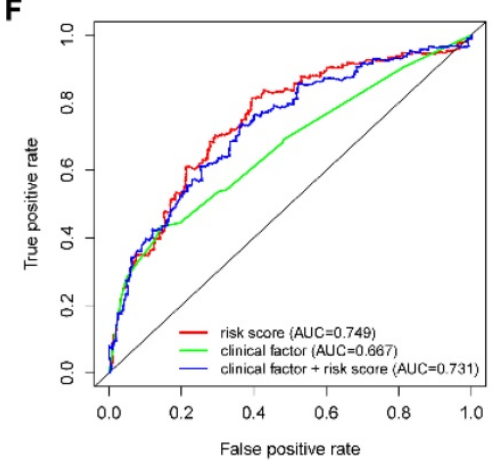

Figure 4. ROC analysis of clinical factors and the risk score. Calculated AUCs for risk score, age, stage, histological type, and grade of the total survival risk score according to the ROC curve at (A) one year, (B) three years, and (C) five years. Calculated AUCs for risk score and combined clinical factors of the total survival risk score according to the ROC curve at $(D)$ one year, $(E)$ three years, and $(F)$ five years. 


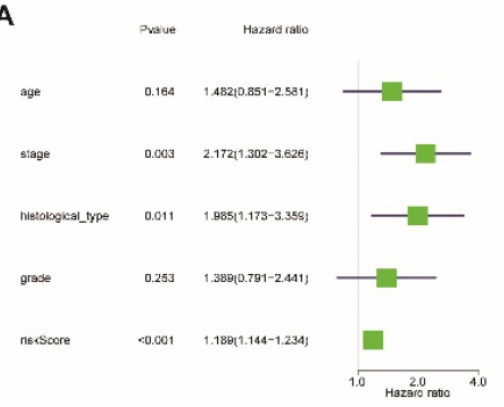

B

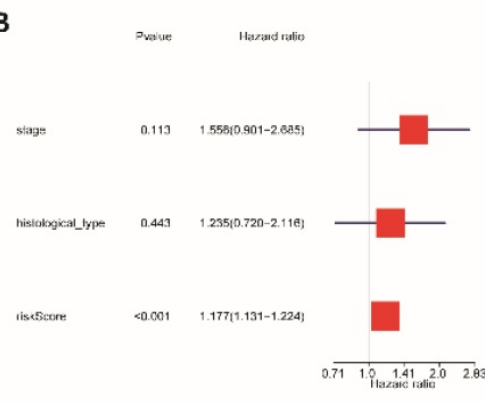

C

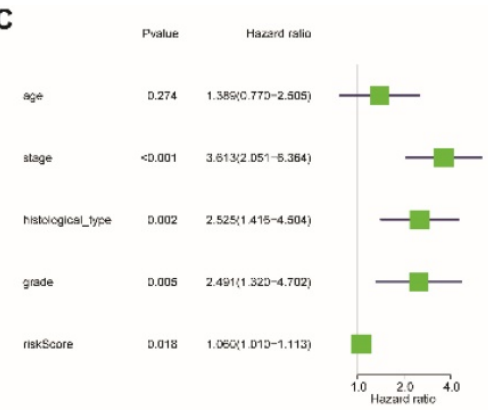

D

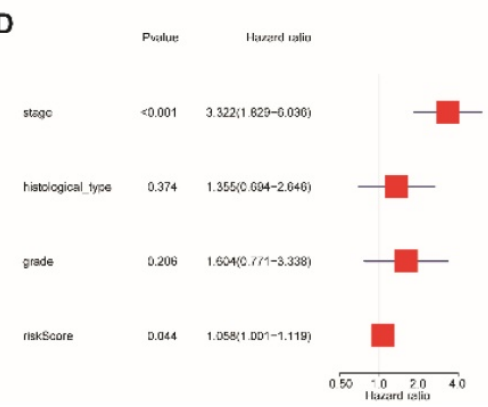

E

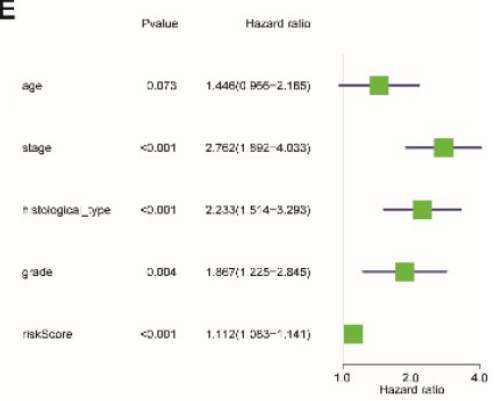

$\mathbf{F}$

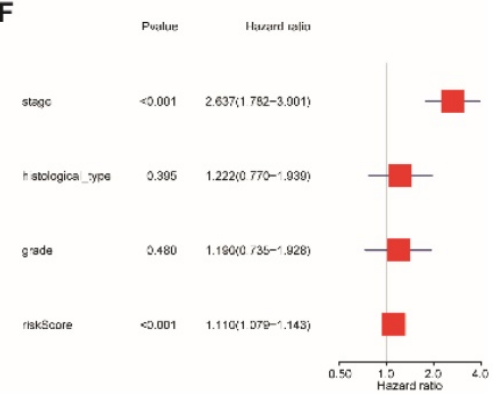

Figure 5. Cox regression analysis was used to assess the independent prognostic value of the risk score. Univariate Cox regression analysis of age, stage, histological type, grade, and risk score in the (A) training cohort, (B) testing cohort, and (C) entire cohort. The multivariate Cox regression analysis of age, stage, histological type, grade, and risk score in the $(D)$ training cohort, $(E)$ testing cohort, and $(F)$ entire cohort.

We next built a nomogram to indicate 1-, 2-, 3-, and 5-year DFS among EnCa patients using stage and risk score, which was identified as an independent prognostic factor for EnCa patients (Fig. 6A). Calibration plots showed that the mortality estimated by the nomogram was close to the actual mortality (Fig. 6B). All findings suggested that the 13-lncRNA prognostic signature for EnCa was highly reliable.

\section{Different immune status between high- and low-risk groups}

Given that the 13-IncRNA signature was developed using immune-related lncRNAs, we further evaluated whether the novel signature was correlated with the expression of immune checkpoints. The results showed that patients with low risk tended to express higher CTLA-4 and PD-1 levels $(P<0.05$, Fig. S9A). In addition, the risk score was negatively correlated with the expression of CTLA-4 and PD- $1(P<0.05$, Fig. S9B-D).

Moreover, to assess the correlations between risk score and tumor immune cell infiltration, CIBERSORT was applied to estimate the proportion of the 22 immune cells by using transcriptome data in each EnCa sample. We appraised that resting CD4+ memory $\mathrm{T}$ cells, M0 macrophages, and activated master cells were positively correlated with the 13-lncRNA risk score, while plasma cells, CD8+ $\mathrm{T}$-cells, activated CD4+ memory $\mathrm{T}$ cells, regulatory $\mathrm{T}$ cells, activated dendritic cells, and resting master cells were negatively correlated with the 13-lncRNA risk score $(P<0.05$, Fig. $7 A-B)$. In addition, the top 3 immune cells that were most positively and negatively associated with the risk score $(P<0.05)$ are shown in Fig. 7C-H.

\section{Mutational loading in high- and low-risk groups}

We next investigated whether EnCa with a high-risk score was associated with tumor mutation burden (TMB). The altered landscapes in EnCa with high- or low-risk scores are shown in Fig. 8A-B. Ten genes were mutated in $>20 \%$ of samples with high-risk scores: PTEN (53\%), TP53 (45\%), PIK3CA (44\%), ARID1A (38\%), TTN (35\%), PIK3R1 (28\%), CSMD3 (22\%), KMT2D (21\%), CTCF (20\%), and RYR2 $(20 \%)$. Ten genes were mutated in $>30 \%$ of samples with low-risk scores: PTEN (78\%), PIK3CA (56\%), ARID1A (54\%), TTN (43\%), CTNNB1 (34\%), CTCF (34\%), KMT2D (34\%), PIK3R1 (32\%), MUC16 (31\%), and MUC5B (30\%). Specifically, the rates of PTEN mutation, PIK3CA mutation, ARID1A mutation, TTN mutation, PIK3R1 mutation, KMY2D mutation, and CTCF mutation were lower in EnCa with high-risk scores than in EnCa with low-risk scores (Fig. 8A-B). In addition, patients with low-risk scores tended to have a higher TMB than those with high-risk scores $(P=0.003$, Fig. $8 \mathrm{C})$. Moreover, the risk score was negatively correlated with TMB $(P<0.001$, Fig. $8 D)$. Furthermore, we found that high TMB was associated with poorer DFS ( $P=0.009$, Fig. $8 \mathrm{E})$. 
A

Points

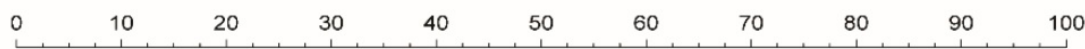

stage

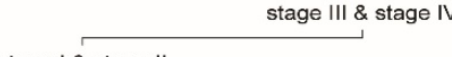

stage | \& stage ||

riskScore

\begin{tabular}{llllllll}
\hline 0 & 5 & 10 & 15 & 20 & 25 & 30 & 35 \\
\hline
\end{tabular}

Total Points

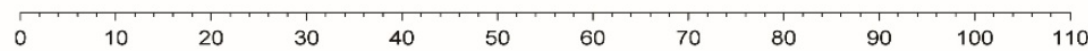

1-year DFS

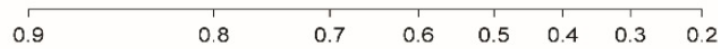

2-year DFS

$\begin{array}{llllllllll}0.9 & 0.8 & 0.7 & 0.6 & 0.5 & 0.4 & 0.3 & 0.2 & 0.1 & 0.05\end{array}$

3-year DFS

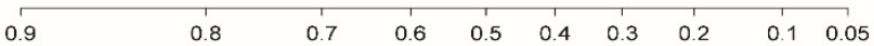

5-year DFS

\begin{tabular}{lllllllll}
\hline 0.8 & 0.7 & 0.6 & 0.5 & 0.4 & 0.3 & 0.2 & 0.1 & 0.05
\end{tabular}

B
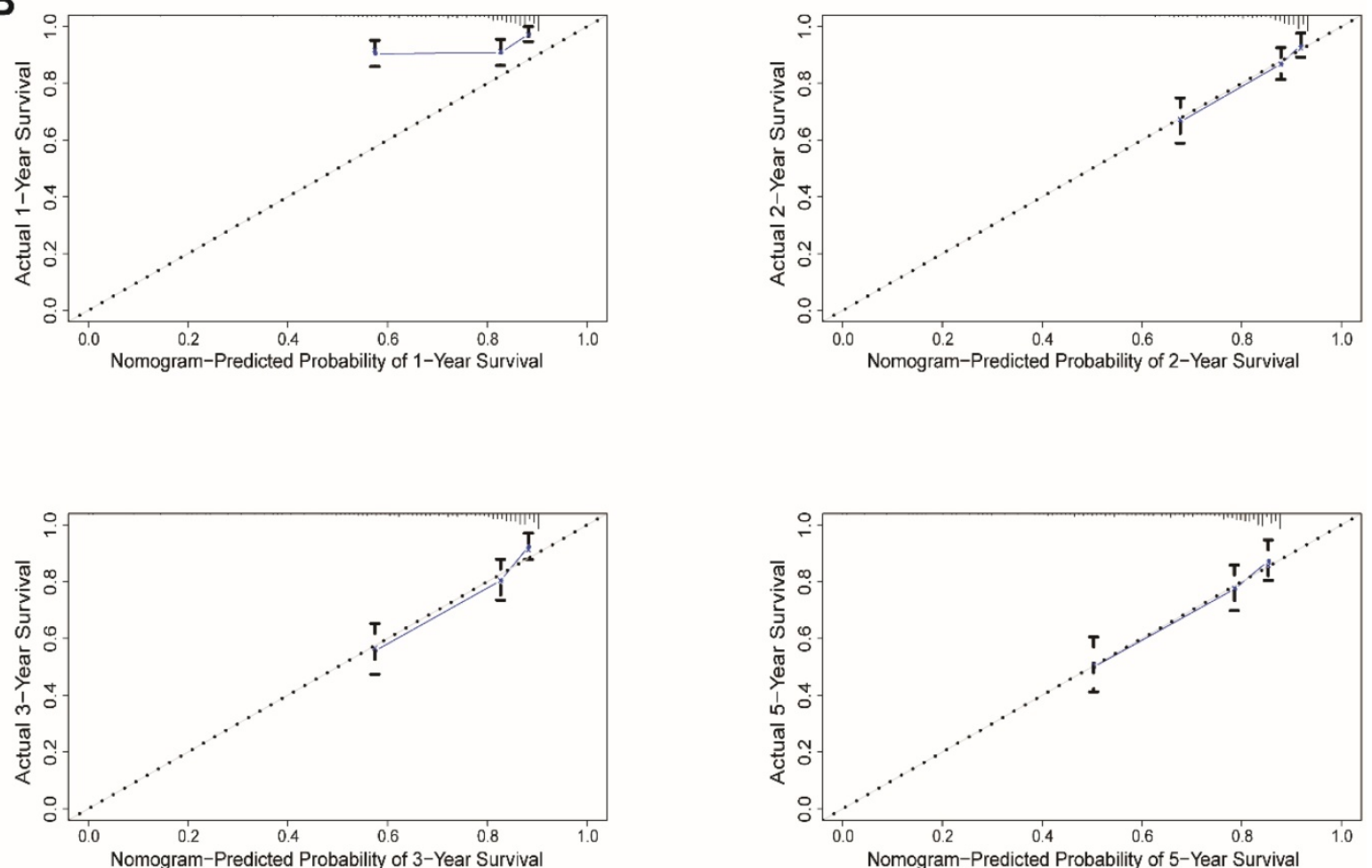

Figure 6. Construction of a nomogram for survival prediction. (A) The nomogram combining the signature with clinicopathological features. (B) Calibration plot showing that nomogram-predicted survival probabilities corresponded closely to the actual observed proportions.

\section{Response to immunotherapy and chemotherapy in high- and low-risk groups}

We also assessed the association between IPS and our immune signature score. The IPS, PD1-PDL1-PD-L2-IPS, CTLA4-IPS, and PD1-PD-L1-PD-L2-
CTLA4-IPS scores were calculated to evaluate the potential for patients to be placed on immunotherapy. The results showed that the PD1-PD-L1-PD-L2CTLA4-IPS score was notably higher in the low-risk group $(P<0.05)$, while the other three scores had no significant association with the risk score (Fig. S10). 
A

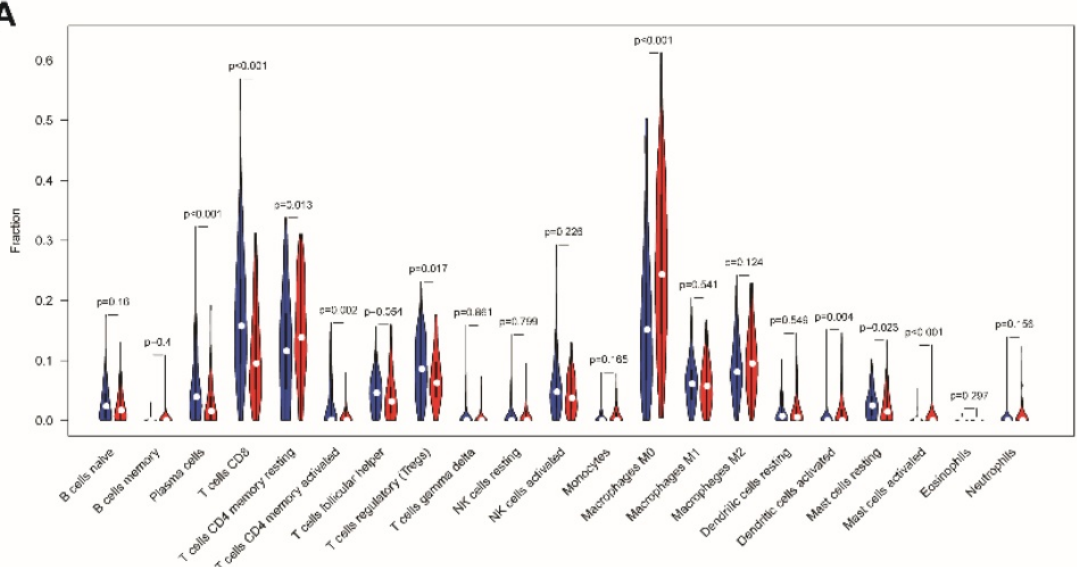

C

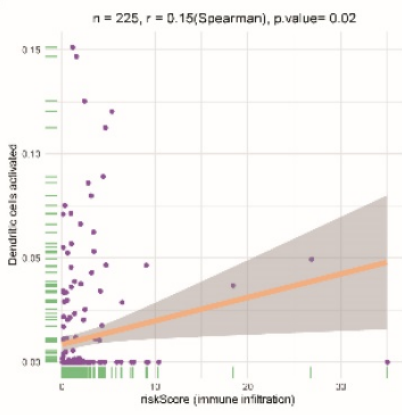

$\mathbf{F}$

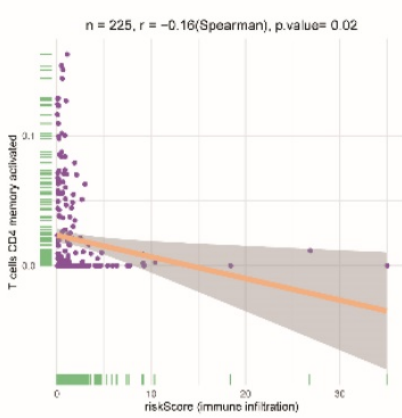

D

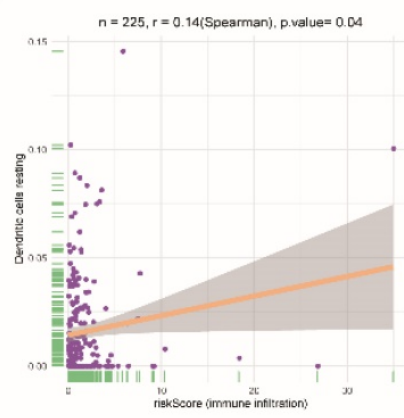

G

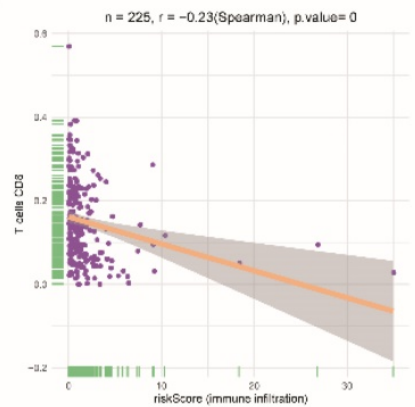

B

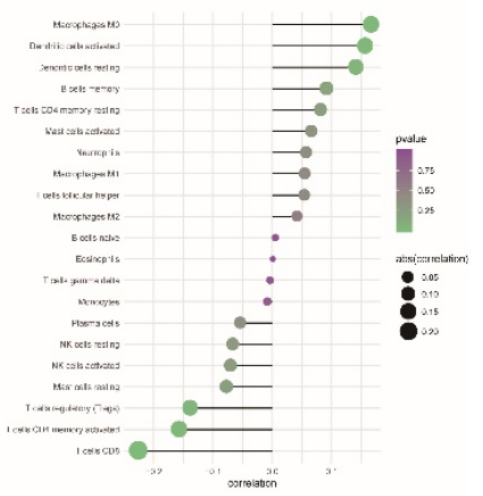

E

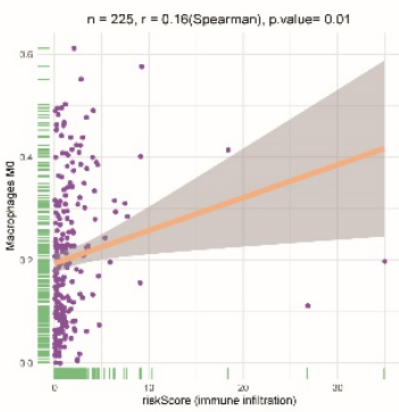

H

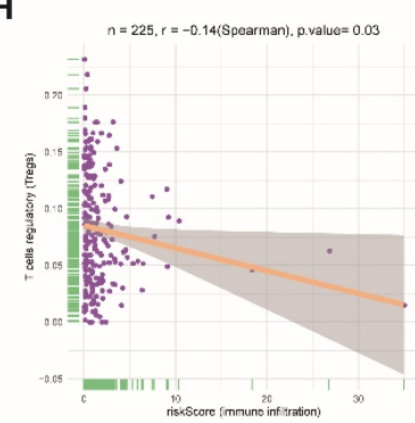

Figure 7. Association between immune cell infiltration and the immune-related risk signature. (A) The violin plot represents immune cell infiltration in the high- and low-risk groups. (B) The Bubble plot represents the correlation between immune cell infiltration and the immune-related risk score. Positive correlation between (C) activated dendritic cells, (D) resting dendritic cells, or (E) M0 macrophage infiltration and the immune-related risk score. Negative correlation between (F) activated CD4+ memory T cells, (G) $\mathrm{CD} 8+\mathrm{T}$ cells, or $(\mathrm{H})$ regulatory $\mathrm{T}$ cell infiltration and the immune-related risk score.

Because chemotherapy is the standard therapeutic strategy for EnCa patients, we assessed the response of patients in these two groups to several common chemo drugs. We estimated the IC50 for each sample in the TCGA dataset based on the predictive model of these chemo drugs. We observed a significant difference in the estimated IC50 between the low- and high-risk groups for gemcitabine, vinblastine, and vorinostat $(P<0.05)$, indicating that patients with high risk could be more resistant to these chemotherapies, which explains the poor prognosis of patients in the high-risk group (Fig. S11).

\section{Discussion}

EnCa is a common gynecological malignancy that poses a great threat to women's lives. Although more than $70 \%$ of cases can be diagnosed at the early stage, approximately $30 \%$ of patients have regional and/or distant metastasis when first seen in the clinic $[20,21]$. Unfortunately, the prognosis of EnCa patients with advanced-stage disease is often unsatisfactory. The prognostic evaluation of EnCa has always been a hot topic for concerned scholars. Construction of scoring models will contribute to quantification of the prognostic evaluation criteria, and an increasing number of studies are successfully establishing precedents in this regard [22-24]. 
A

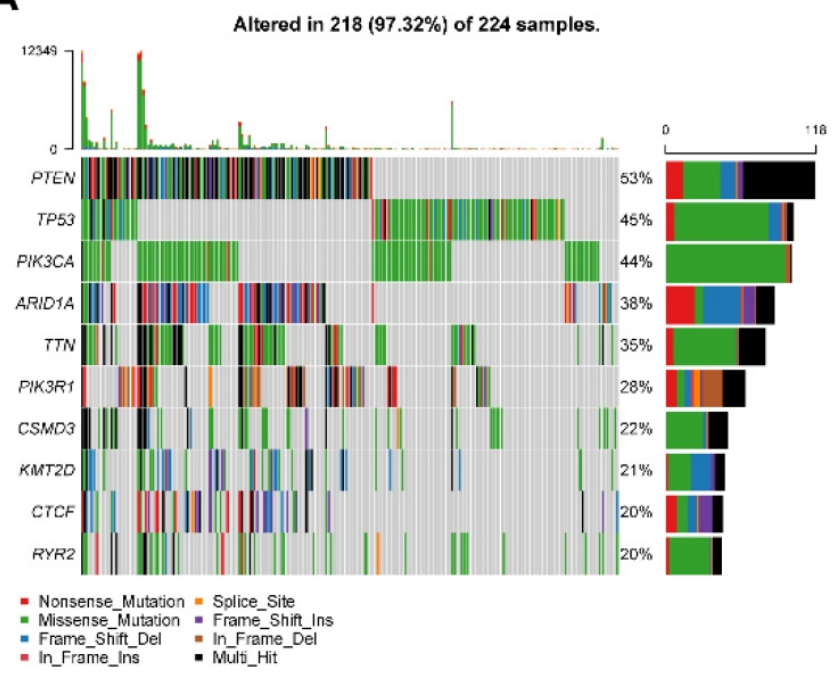

B

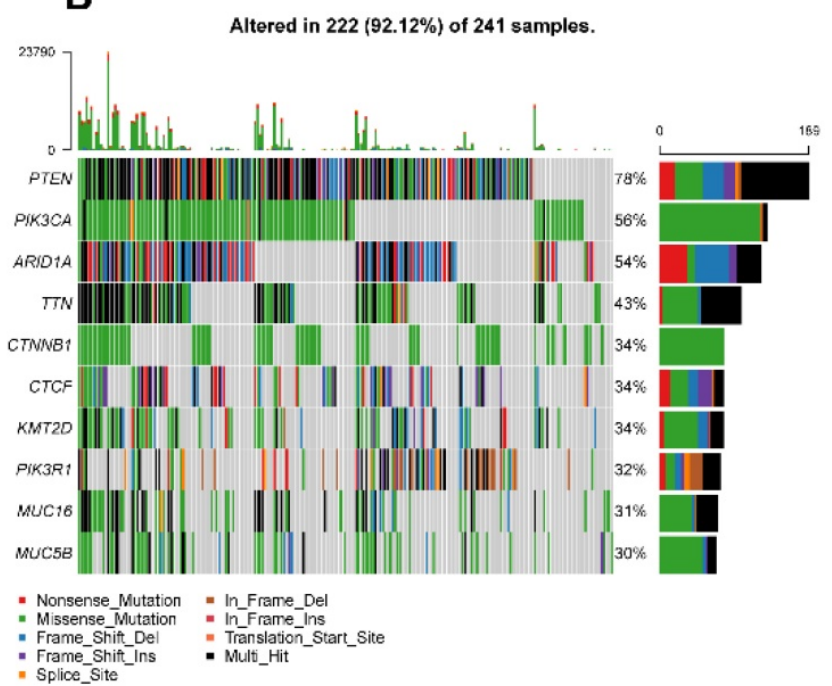

C

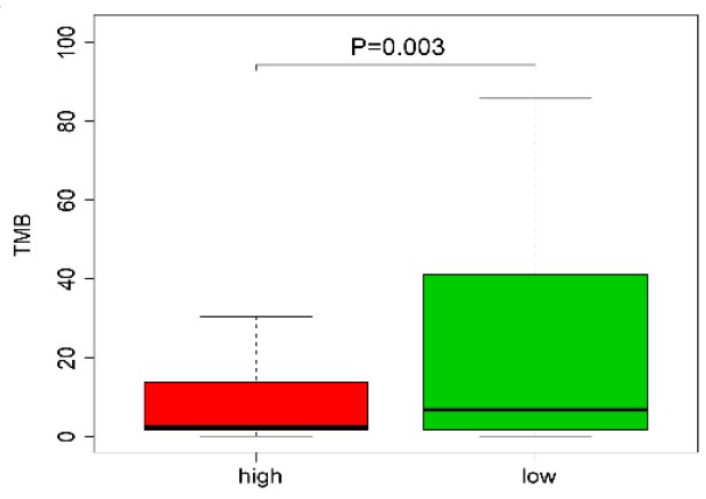

D

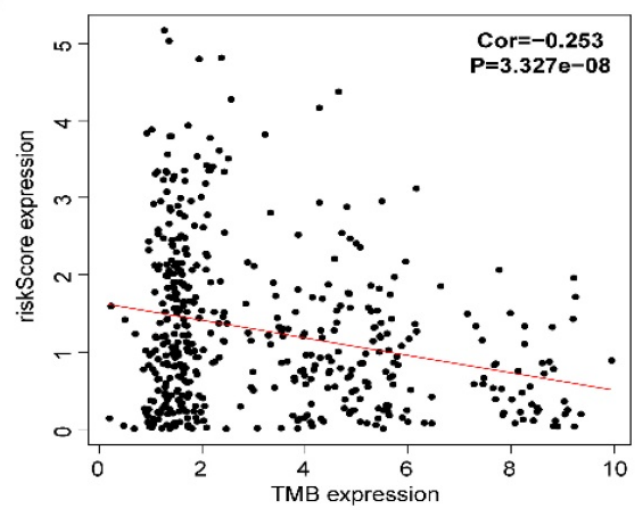

E

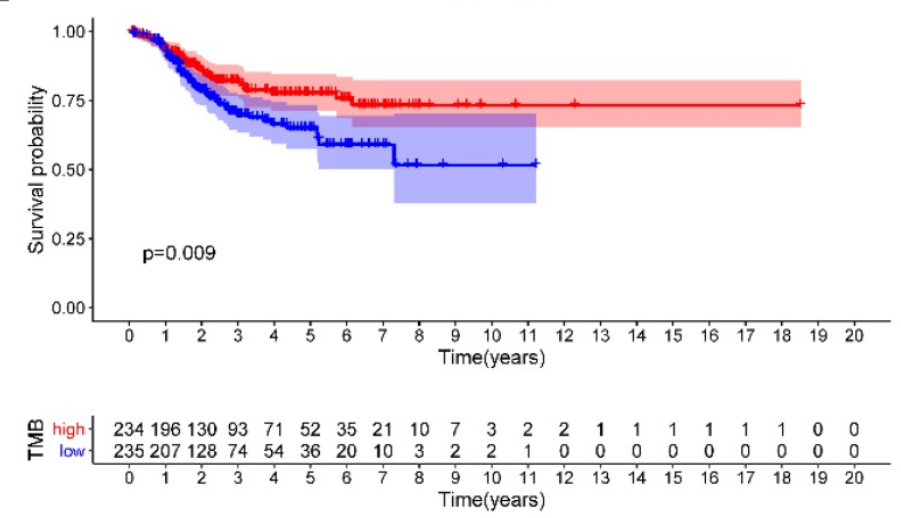

Figure 8. Alteration landscape for high- and low-risk EnCa samples in the TCGA cohort. (A, B) The rates of PTEN mutation, PIK3CA mutation, ARIDIA mutation, TTN mutation, and PIK3R1 mutation in EnCa with a high-risk score were lower than those with EnCa with a low-risk score. (C) EnCa patients with high-risk scores had a heavier tumor mutation burden than with those with low-risk scores. (D) Negative correlation between tumor mutation burden and the immune-related risk score. (E) Kaplan-Meier curve analysis of DFS of EnCa patients with different tumor mutation burdens.

In the current research, a series of systematic bioinformatics analyses were conducted, ultimately leading to the development of a novel 13-lncRNA signature associated with the clinical outcome of EnCa patients; this signature included AL080317.2,
ERICH6-AS1, AC016877.3, MCCC1-AS1, AC120053.1, AC138932.5, ZNF433-AS1, SCARNA9, DBH-AS1, AL157932.1, AC073046.1, POC1B-AS1, and AP003419.3. The novel signature constructed based on the expression of these 13 immune-related 
lncRNAs allowed us to distinguish samples with different risks with relatively high accuracy. In the training set, EnCa patients in the high-risk group had a shorter DFS than those in the low-risk group, and consistent findings were obtained in the testing and entire sets. Moreover, univariate and multivariate Cox regression analyses confirmed that the signature was an independent factor for predicting DFS in EnCa. All findings confirmed that the 13-lncRNA prognostic model could accurately predict the DFS of patients with EnCa.

Among the 13 immune-related lncRNAs, ERICH6-AS1, MCCC1-AS1, AC120053.1, AC138932.5, ZNF433-AS1， DBH-AS1，AL157932.1，AC073046.1, POC1B-AS1, and AP003419.3 were risk-associated lncRNAs, while AC016877.3, AL080317.2, and SCARNA9 were protective lncRNAs. LncRNAmRNA coexpression analysis was conducted to evaluate the functions of related lncRNAs. Several lncRNAs have been identified as functional genes in cancers. For example, DBH-AS1 may act as an oncogene, and downregulation of DBH-AS1 predicts better prognosis and suppresses osteosarcoma progression by inhibiting the PI3K/AKT pathway [25]. In addition, DBH-AS1 can accelerate the tumorigenesis and development of hepatocellular carcinoma by sponging miR-138 and regulating the FAK/Src/ERK pathway [26]. Wang et al. developed of a multi-RNA-type-based model for recurrence-free survival (RFS) evaluations of patients with EnCa [27]. In addition, we also determined the expression of lncRNAs in normal and tumor tissues. Four lncRNAs were upregulated, while three lncRNAs were downregulated. Therefore, the 13 immune-related lncRNAs might be potential therapeutic targets because of the dysregulation of lncRNAs.

In recent years, immunotherapy has been considered a novel therapeutic strategy for EnCa [4, 5, 28]. The tumor microenvironment (TME), which contains extracellular matrix, fibroblasts, endothelial cells, and multiple immune cells, plays a critical role in tumor progression, immune escape and responses to therapies, especially immunotherapies [29]. Thus, identification of a single gene and/or gene signature that correlates with immune cell infiltration is essential for the assessment of responses to immunotherapies.

As an important part of the current research, we evaluated the association between the expression of 13 hub lncRNAs and immune cell infiltration. Most lncRNAs were correlated with specific immune cell infiltration; among them, ERICH6-AS1 had the strongest correlation with immune cell infiltration. In addition, the risk model based on the expression of 13 immune-related lncRNAs was also significantly associated with different immune statuses in EnCa. $\mathrm{TMB}$ is an emerging biomarker for assessing the response to PD-1/PD-L1 inhibitors [30, 31]. We also evaluated the correlation between the risk score of the prognosis signature and TMB. Patients with low risk tended to have a higher TMB than those with high risk, and the risk score was negatively correlated with TMB. Overall, the 13-lncRNA signature might serve as a biomarker to assess the feasibility of immunotherapies.

Although immunotherapy could be a novel therapeutic strategy, EnCa is usually treated with a combined regimen of surgery and chemotherapy [32, 33]. Using the GDSC database, we found that EnCa patients with low risk could be more sensitive to commonly used chemotherapies, including gemcitabine, vinblastine, and vorinostat, than those with high risk, which demonstrated that low-risk patients may benefit from this combination of chemotherapy.

However, the current research has several unavoidable limitations. Most important of all, the research is based on bioinformatics analysis, and there is no external EnCa data as a validation set; secondly, there were no recruited cohorts for verification of prognostic value of the signature in $\mathrm{EnCa}$ and response to immunotherapy and chemotherapy in high- and low-risk groups. Whatever, we will pay more attention to emerging external data for further verifying our model in the future.

\section{Conclusions}

In conclusion, we identified immune-related lncRNAs in EnCa and developed a 13-lncRNA signature that has significant prognostic value for patients with EnCa. Furthermore, high- and low-risk groups, which were divided based on the median risk score, displayed different immune statuses and responses to immunotherapy and chemotherapy.

\section{Abbreviations}

EnCa: endometrial carcinoma; FIGO: International Federation of Gynecology and Obstetrics; LncRNAs: long noncoding RNAs; PD-L1: programmed cell death 1 ligand 1; DFS: disease-free survival; TMB: tumor mutation burden; GSEA: gene set enrichment analysis; MAF: mutation annotation format; IPS: immunophenoscore; TCIA: Cancer Immunome Atlas; GDSC: Genomics of Drug Sensitivity in Cancer; IC50: half-maximal inhibitory concentration; RFS: recurrence-free survival; TME: tumor microenvironment. 


\section{Supplementary Material}

Supplementary figures and tables.

http://www.ijbs.com/v17p0448s1.pdf

\section{Acknowledgements}

\section{Author contribution}

Jinhui Liu: Conceptualization, Methodology, Data curation, Visualization, Formal analysis, Writing-Original draft preparation. Jie Mei: Methodology, Formal analysis, Visualization, Writing-Original draft preparation. Yichun Wang:

\section{Competing Interests}

The authors have declared that no competing interest exists.

\section{References}

1. Siegel RL, Miller KD, Jemal A. Cancer Statistics, 2017. CA Cancer J Clin. 2017; 67: 7-30

2. Ferlay J, Soerjomataram I, Dikshit R, Eser S, Mathers C, Rebelo M, et al. Cancer incidence and mortality worldwide: sources, methods and major patterns in GLOBOCAN 2012. Int J Cancer. 2015; 136: E359-86.

3. Mang C, Birkenmaier A, Cathomas G, Humburg J. Endometrioid endometrial adenocarcinoma: an increase of G3 cancers? Arch Gynecol Obstet. 2017; 295: $1435-40$

4. Di Tucci C, Capone C, Galati G, Iacobelli V, Schiavi MC, Di Donato V, et al. Immunotherapy in endometrial cancer: new scenarios on the horizon. J Gynecol Oncol. 2019; 30: e46.

5. Green AK, Feinberg J, Makker V. A Review of Immune Checkpoint Blockade Therapy in Endometrial Cancer. Am Soc Clin Oncol Educ Book. 2020; 40: 1-7.

6. Mei J, Hao L, Wang H, Xu R, Liu Y, Zhu Y, et al. Systematic characterization of non-coding RNAs in triple-negative breast cancer. Cell Proliferation. 2020.

7. Liu H, Wan J, Chu J. Long non-coding RNAs and endometrial cancer. Biomed Pharmacother. 2019; 119: 109396.

8. Xing TR, Chen P, Wu JM, Gao LL, Yang W, Cheng Y, et al. UPF1 Participates in the Progression of Endometrial Cancer by Inhibiting the Expression of IncRNA PVT1. Onco Targets Ther. 2020; 13: 2103-14.

9. Wang J, Li Z, Wang X, Ding Y, Li N. The tumor suppressive effect of long non-coding RNA FRMD6-AS2 in uteri corpus endometrial carcinoma. Life Sci. 2020; 243: 117254

10. Xu S, Wang Q, Kang Y, Liu J, Yin Y, Liu L, et al. Long noncoding RNAs control the modulation of immune checkpoint molecules in cancer. Cancer Immunol Res. 2020.

11. Luo Y, Yang J, Yu J, Liu X, Yu C, Hu J, et al. Long Non-coding RNAs: Emerging Roles in the Immunosuppressive Tumor Microenvironment. Front Oncol. 2020; 10: 48

12. $\mathrm{Ma} \mathrm{H}$, Chang $\mathrm{H}$, Yang $\mathrm{W}, \mathrm{Lu} \mathrm{Y}, \mathrm{Hu}$ J, Jin S. A novel IFNalpha-induced long noncoding RNA negatively regulates immunosuppression by interrupting H3K27 acetylation in head and neck squamous cell carcinoma. Mol Cancer. 2020; 19: 4.

13. Wang W, Zhao Z, Yang F, Wang H, Wu F, Liang T, et al. An immune-related IncRNA signature for patients with anaplastic gliomas. J Neurooncol. 2018; 136: 263-71.

14. Song J, Xu Q, Zhang H, Yin X, Zhu C, Zhao K, et al. Five key lncRNAs considered as prognostic targets for predicting pancreatic ductal adenocarcinoma. J Cell Biochem. 2018; 119: 4559-69.

15. Newman AM, Liu CL, Green MR, Gentles AJ, Feng W, Xu Y, et al. Robust enumeration of cell subsets from tissue expression profiles. Nat Methods. 2015; $12:$ 453-7.

16. Subramanian A, Kuehn H, Gould J, Tamayo P, Mesirov JP. GSEA-P: a desktop application for Gene Set Enrichment Analysis. Bioinformatics. 2007; 23: 3251-3.

17. Mayakonda A, Lin DC, Assenov Y, Plass C, Koeffler HP. Maftools: efficient and comprehensive analysis of somatic variants in cancer. Genome research. 2018; 28 : 1747-56.

18. Charoentong P, Finotello F, Angelova M, Mayer C, Efremova M, Rieder D, et al. Pan-cancer Immunogenomic Analyses Reveal Genotype-Immunophenotype Relationships and Predictors of Response to Checkpoint Blockade. Cell Rep. 2017; 18: 248-62.

19. Geeleher P, Cox NJ, Huang RS. Clinical drug response can be predicted using baseline gene expression levels and in vitro drug sensitivity in cell lines. Genome Biol. 2014; 15: R47.

20. Banno K, Nogami Y, Kisu I, Yanokura M, Umene K, Masuda K, et al. Candidate biomarkers for genetic and clinicopathological diagnosis of endometrial cancer. Int J Mol Sci. 2013; 14: 12123-37.
Data curation, Visualization, Writing-Original draft preparation. Xucheng Chen: Data curation, Validation. Jiadong Pan: Validation. Laigen Tong: Writing-Review \& Editing, Project administration. Yan Zhang: Writing-Review \& Editing, Supervision, Project administration, Funding acquisition.

\section{Funding}

This work was founded by the Wuxi Science and Technology Bureau Project (No. CSE31N1720) and the Jiangsu Provincial Six Talent Peaks Project (No. YY-124).

21. Liu J, Li C, Jiang Y, Wan Y, Zhou S, Cheng W. Tumor-suppressor role of miR-139-5p in endometrial cancer. Cancer Cell Int. 2018; 18: 51.

22. Tang J, Ma W, Luo L. Establishment of the Prognosis Predicting Signature for Endometrial Cancer Patient. Med Sci Monit. 2019; 25: 8248-59.

23. Gao L, Nie X, Zheng M, Li X, Guo Q, Liu J, et al. TMEFF2 is a novel prognosis signature and target for endometrial carcinoma. Life Sci. 2020; 243: 116910.

24. Watanabe T, Honma R, Kojima M, Nomura S, Furukawa S, Soeda S, et al. Prediction of lymphovascular space invasion in endometrial cancer using the 55-gene signature selected by DNA microarray analysis. PLoS One. 2019; 14: $\mathrm{e} 0223178$.

25. Liu ZB, Wang JA, Lv RQ. Downregulation of long non-coding RNA DBH-AS1 inhibits osteosarcoma progression by PI3K-AKT signaling pathways and indicates good prognosis. Eur Rev Med Pharmacol Sci. 2019; 23: 1418-27.

26. Bao J, Chen $X$, Hou $Y$, Kang G, Li $O, X u$ Y. LncRNA DBH-AS1 facilitates the tumorigenesis of hepatocellular carcinoma by targeting miR-138 via FAK/ Src/ERK pathway. Biomed Pharmacother. 2018; 107: 824-33.

27. Wang P, Zeng Z, Shen X, Tian X, Ye Q. Identification of a Multi-RNA-TypeBased Signature for Recurrence-Free Survival Prediction in Patients with Uterine Corpus Endometrial Carcinoma. DNA Cell Biol. 2020; 39: 615-30.

28. Brooks RA, Fleming GF, Lastra RR, Lee NK, Moroney JW, Son $\mathrm{CH}$, et al. Current recommendations and recent progress in endometrial cancer. $\mathrm{CA}$ Cancer J Clin. 2019; 69: 258-79.

29. Chen $\mathrm{P}$, Yang $\mathrm{Y}$, Zhang $\mathrm{Y}$, Jiang S, Li X, Wan J. Identification of prognostic immune-related genes in the tumor microenvironment of endometrial cancer. Aging (Albany NY). 2020; 12: 3371-87.

30. Fumet JD, Truntzer C, Yarchoan M, Ghiringhelli F. Tumour mutational burden as a biomarker for immunotherapy: Current data and emerging concepts. Eur J Cancer. 2020; 131: 40-50.

31. Choucair K, Morand S, Stanbery L, Edelman G, Dworkin L, Nemunaitis J. TMB: a promising immune-response biomarker, and potential spearhead in advancing targeted therapy trials. Cancer Gene Ther. 2020.

32. Guo F, Zhang H, Jia Z, Cui M, Tian J. Chemoresistance and targeting of growth factors/cytokines signalling pathways: towards the development of effective therapeutic strategy for endometrial cancer. Am J Cancer Res. 2018; 8: 1317-31.

33. Pan K, Gong J, Huynh K, Cristea M. Current Systemic Treatment Landscape of Advanced Gynecologic Malignancies. Target Oncol. 2019; 14: 269-83. 